\title{
Design an Asymmetrical Three-Beam Laser Interference Lithography for Fabricating Micro- and Nano-Structures
}

\author{
Litong Dong ${ }^{1,2}$, Ziang Zhang ${ }^{3}$, Zuobin Wang ${ }^{1,2^{*}}$, Dayou $\mathrm{Li}^{2 *}$, and Mengnan Liu ${ }^{1}$ \\ ${ }^{1}$ International Research Centre for Nano Handling and Manufacturing of China, Changchun Uni- \\ versity of Science and Technology, Changchun 130022, China \\ ${ }^{2}$ IRAC \& JR3CN, University of Bedfordshire, Luton LU1 3JU, UK \\ ${ }^{3}$ Changchun Observatory, National Astronomical Observatories, CAS, Changchun 130117, China \\ *E-mail:wangz@cust.edu.cn,dayou.li@beds.ac.uk
}

\begin{abstract}
Multi-beam laser interference lithography (LIL) has become one of the most important techniques and shown significant advantages in the fabrication of micro- and nano-structures. Controlling intensity ratio of optical distributions is a key issue in LIL for fabricating micro- and nano-structures. This paper presents an asymmetrical three-beam LIL system which effectively improves the intensity ratio of optical distributions. Comparing with the symmetrical three-beam interference, the asymmetrical three-beam LIL achieved the high intensity ratio of optical distribution when producing the similar interference pattern. In addition, this system also avoids modulation patterns in multi-beam LIL systems and reduces the difficulty of actual LIL processing. A fast Fourier Transform (FFT) analysis used to study the pattern distributions of the asymmetrical three-beam interference from frequency spectra which shows that the pattern with a high-intensity array can be obtained by adjusting the parameter settings of incident laser beams. The asymmetrical three-beam LIL system was verified through fabricating patterns. The experimental results are in good agreement with the theoretical analyses.
\end{abstract}

DOI: $10.2961 /$ jlmn.2020.02.2002

Keywords: laser interference lithography, polarization, azimuth angle, fast Fourier Transform

\section{Introduction}

Periodic micro- and nano- structures have attracted much attention due to their natural advantages in adjusting diffraction, wettability, anti-reflection, and structural coloration [1-3]. Typical fabrications of periodic and quasi-periodic structures include metal-assisted chemical etching (MACE) [4], e-beam lithography (EBL) [5], ion beam lithography (IBL) [6], scanning probe lithography (SPL) [7], and laser-induced periodic surface structures (LIPSS) [8]. However, low throughput, high cost, and limited availability constrain scalable producing functional structures using the aforementioned approaches. Femtosecond lasers are commonly used to prepare micro- and nano-structures with the advantage of their minimal thermal effect [9-10]. But the technique essentially scans an object point-by-point by a focused femtosecond laser, which limits its processing efficiency. Laser interference lithography (LIL) as an optical maskless lithography to record periodic patterns emerged as alternatives to traditional optical lithography for fabrication at low cost, large-scale and with high throughput. Depending on the morphologies of functional surfaces, periodic structures can be fabricated by designing appropriate configurations of multi-beam LIL, leading to changes in specified properties of the material surfaces.

Appropriate configuration of multi-beam LIL can be used to generate ordered structural morphologies, which is a major challenge for obtaining specified functional artificial materials. For example, two-beam LIL is an effective method for the preparation of periodic one-dimensional structures, which can easily control the period of structures from nanometers to micrometers for the purpose of adjusting structural colours [11-12] and wetting anisotropy [13-14]. Three-beam LIL can effectively form highly ordered superhydrophobic surfaces on silicon wafers [16-17]. Four-beam LIL was often used to manufacture multifunctional structures [18-20] and 3D photonic crystals [21-22]. Biomimetic structures were directly produced using six-beam LIL [23]. LIL is a versatile tool to make functional micro-and nanostructures. Graus et al. [24] reported a direct laser interference patterning for fabricating periodic magnetic nanostructures on $\mathrm{Fe}_{60} \mathrm{Al}_{40}$ films. Wang et al. [25] presented a laserinterference-controlled electrochemical deposition method for direct fabrication of periodically micropatterned magnetite $\left(\mathrm{Fe}_{3} \mathrm{O}_{4}\right)$ nanoparticles. Patterns of structures rely on the parameters of coherent laser beams which controlled the optical field distribution in LIL. Studying the theoretical model of light field distributions can accurately realize designed periodic structures and control their parameters, including the period, shape and distribution of the structures, which improves processing efficiency of fabricating functional materials [26-29]. In the previous work, we have discussed the effect of four-beam laser interference parameters on forming patterns and the modulation phenomenon [19, 30-31]. However, there are still many issues worthy of discussion and research in multi-beam LIL, especially the intensity ratio of interference fields. On the other hand, the main difficulties in the construction of the spatial structure of the optical setup are to determine the positions of incident beams, including azimuth angles, incident angles, and polarization directions. Therefore, a simplified optics design of multi-beam LIL is important to implement industrial strategies. 
In this paper, an asymmetrical three-beam LIL is presented for fabricating micro- and nano-structures. This design can form a higher intensity ratio of optical field distributions when producing a similar interference pattern as that of the symmetrical three-beam interference. The optical field distribution of multi-beam interference depends on the number of superposed coherent beams and their configuration. In this work, impacts of various parameters of coherent beams on the optical field are studied to effectively control periodic structures with a high duty cycle and consistency via the asymmetrical three-beam LIL system. These theoretical analyses are verified by experimental results, and FFT can analyze and further verify the structure in the frequency domain. The asymmetrical three-beam LIL system is simple, which reduces the difficulty of constructing the actual optical path and improves the control of the pattern of the light field.

\section{Optical design and simulation}

For simplicity, all incident beams are considered to be plane waves with the same wavelength and intensity, and the general form of $n$-beam interference can be described as the superposition of electric field vectors of the incident beams $\left(\vec{E}_{1}, \vec{E}_{2}, \ldots\right.$, and $\left.\vec{E}_{n}\right)$.

The electric field distribution of the $n$th plane wave is expressed as:

$$
\vec{E}_{n}=A_{n} \cdot \vec{e}_{n} \cdot \cos \left(\vec{K}_{n} \cdot \vec{r}-\omega \cdot t+\varphi_{0 n}\right),
$$

where $A_{n}$ is the amplitude, $\vec{e}_{n}$ is the unit polarization vector, $\vec{K}_{n}$ is the wave vector, $\vec{r}$ is the position vector in the propagation direction, $\omega$ is the frequency, and $\varphi_{0 n}$ is the initial phase.

In Eq. (1), $\vec{r}$ and $\vec{K}_{n}$ and $\vec{e}_{n}$ can be obtained by:

$\vec{r}=(x, y, z)$,

$\vec{K}_{n}=\left(k \cdot \sin \theta_{n} \cdot \cos \phi_{n}, \quad k \cdot \sin \theta_{n} \cdot \sin \phi_{n}, \quad-k \cdot \cos \theta_{n}\right)$,

$\vec{e}_{n}=\left(\begin{array}{c}-\cos \theta_{n} \cdot \cos \phi_{n} \cdot \cos \psi_{n}+\sin \phi_{n} \cdot \sin \psi_{n}, \\ -\cos \theta_{n} \cdot \sin \phi_{n} \cdot \cos \psi_{n}-\cos \phi_{n} \cdot \sin \psi_{n}, \\ -\sin \theta_{n} \cdot \cos \psi_{n}\end{array}\right)$, where $k$ is the wave number and $k=2 \pi / \lambda$ ( $\lambda$ is the wavelength of incident beams), $\theta_{n}, \phi_{n}$ and $\psi_{n}$ are the incident angle, the azimuthal angle, and the polarization angle of $n$th incident beam, respectively. It can be found from Eq. (3) that a wave vector is independent of the polarization mode of coherent beams.

The intensity distribution of multi-beam interference pattern can be written as [30]:

$$
\begin{aligned}
& I(\vec{r})=\left|\sum_{j=1}^{N} E_{j}\right|^{2} \\
& =\sum_{j=1}^{N} E_{0 j}^{2}+2 \sum_{i<j} E_{0 i} E_{0 j} e_{i j} \cos \left[\left(\vec{K}_{i}-\vec{K}_{j}\right) \cdot \vec{r}+\varphi_{0 i}-\varphi_{0 j}\right] .
\end{aligned}
$$

where $e_{i j}=\vec{e}_{i} \cdot \vec{e}_{j}$.

It can be seen from Eq. (5) that the intensity distribution relies on the spatial position vectors, polarization directions and initial phase difference.

Schematics for three-beam interference configurations are conceptually illustrated in Figure 1. There are four cases of the polarization modes in the symmetrical three-beam interference (Figure 1a), including TE-TE-TE, TM-TM-TM, TE-TM-TE and TM-TE-TM. According to Eq. (4), Table 1 shows the polarization modes and the corresponding polarization vectors. (a)

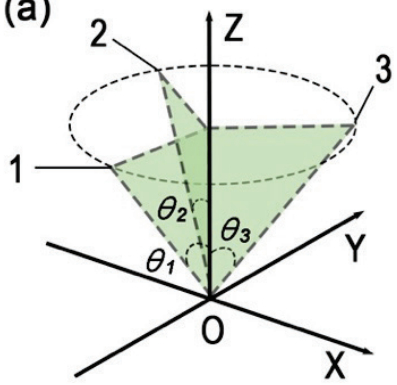

(b)

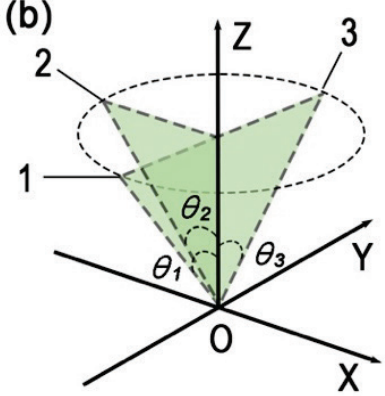

Fig. 1 Two spatial distributions of three-beam interference. (a) The symmetrical three-beam interference configuration with the azimuthal angles of $0^{\circ}, 120^{\circ}$, and $240^{\circ}$; (b) The asymmetrical three-beam interference configuration with the azimuthal angles of $0^{\circ}, 90^{\circ}$, and $180^{\circ}$.

Table 1 Polarization modes and corresponding polarization vectors of the symmetrical three-beam interference.

\begin{tabular}{l}
$\begin{array}{l}\text { Polarization } \\
\text { modes }\end{array}$ \\
\hline $\begin{array}{l}\text { TE-TE-TE } \\
\text { TM-TM-TM }\end{array} \quad \vec{e}_{1-T E}=(0,-1,0), \vec{e}_{2-T E}=\left(\frac{\sqrt{3}}{2}, \frac{1}{2}, 0\right), \vec{e}_{3-T E}=\left(-\frac{\sqrt{3}}{2}, \frac{1}{2}, 0\right)$ \\
TE-TM-TE \\
$\vec{e}_{1-T M}=\left(-\cos \theta_{1}, 0,-\sin \theta_{1}\right), \vec{e}_{2-T M}=\left(\frac{1}{2} \cos \theta_{2},-\frac{\sqrt{3}}{2} \cos \theta_{2},-\sin \theta_{2}\right), \vec{e}_{3-T M}=\left(\frac{1}{2} \cos \theta_{3}, \frac{\sqrt{3}}{2} \cos \theta_{3},-\sin \theta_{3}\right)$ \\
TM-TE-TM \\
$\vec{e}_{1-T M}=\left(-\cos \theta_{1}, 0,-\sin \theta_{1}\right), \vec{e}_{2-T E}=\left(\frac{\sqrt{3}}{2}, \frac{1}{2}, 0\right), \vec{e}_{3-T M}=\left(\frac{1}{2} \cos \theta_{3}, \frac{\sqrt{3}}{2} \cos \theta_{3},-\sin \theta_{3}\right)$
\end{tabular}

It is assumed that amplitudes of each beam are identical, initial phases are 0 , and incident angles are $\theta_{1}=\theta_{2}=\theta_{3}=\theta$. 
The intensity distributions of the symmetrical three-beam interferences in different polarization modes can be expressed as:

$$
\begin{aligned}
& I_{T E-T E-T E}=3 A^{2} \\
& -A^{2} \cdot \cos \left[k \cdot \sin \theta \cdot\left(\frac{3}{2} x-\frac{\sqrt{3}}{2} y\right)\right] \\
& -A^{2} \cdot \cos \left[k \cdot \sin \theta \cdot\left(\frac{3}{2} x+\frac{\sqrt{3}}{2} y\right)\right] \\
& -A^{2} \cdot \cos (\sqrt{3} k \cdot \sin \theta \cdot y) \\
& I_{T M-T M-T M}=3 A^{2} \\
& -\left(\cos { }^{2} \theta-2 \sin ^{2} \theta\right) \cdot A^{2} \cdot \cos \left[k \cdot \sin \theta \cdot\left(\frac{3}{2} x-\frac{\sqrt{3}}{2} y\right)\right] \\
& -\left(\cos { }^{2} \theta-2 \sin ^{2} \theta\right) \cdot A^{2} \cdot \cos \left[k \cdot \sin \theta \cdot\left(\frac{3}{2} x+\frac{\sqrt{3}}{2} y\right)\right] \\
& -\left(\cos { }^{2} \theta-2 \sin ^{2} \theta\right) \cdot A^{2} \cdot \cos (\sqrt{3} k \cdot \sin \theta \cdot y) \\
& I_{T E-T M-T E}=3 A^{2} \\
& +\sqrt{3} \cos \theta \cdot A^{2} \cdot \cos (\sqrt{3} k \cdot \sin \theta \cdot y) \\
& +\sqrt{3} \cos \theta \cdot A^{2} \cdot \cos \left[k \cdot \sin \theta \cdot\left(\frac{3}{2} x-\frac{\sqrt{3}}{2} y\right)\right] \\
& -\sqrt{3} \cos \theta \cdot A^{2} \cdot \cos (\sqrt{3} k \cdot \sin \theta \cdot y) \\
& -A^{2} \cdot \cos \left[k \cdot \sin ^{2} \theta \cdot\left(\frac{3}{2} x+\frac{\sqrt{3}}{2} y\right)\right]
\end{aligned}
$$

Table 2 Polarization modes and corresponding polarization vectors of the asymmetrical three-beam interference.

\begin{tabular}{cc}
\hline $\begin{array}{c}\text { Polarization } \\
\text { modes }\end{array}$ & Polarization vectors \\
\hline TE-TM-TE & $\vec{e}_{1-T E}=(0,-1,0), \vec{e}_{2-T M}=\left(0,-\cos \theta_{2},-\sin \theta_{2}\right), \vec{e}_{3-T E}=(0,1,0)$ \\
TM-TE-TM & $\vec{e}_{1-T M}=\left(-\cos \theta_{1}, 0,-\sin \theta_{1}\right), \vec{e}_{2-T E}=(1,0,0), \vec{e}_{3-T M}=\left(\cos \theta_{3}, 0,-\sin \theta_{3}\right)$ \\
TM-TM-TE & $\vec{e}_{1-T M}=\left(-\cos \theta_{1}, 0,-\sin \theta_{1}\right), \vec{e}_{2-T M}=\left(0,-\cos \theta_{2},-\sin \theta_{2}\right), \vec{e}_{3-T E}=(0,1,0)$ \\
\hline
\end{tabular}

The interference intensity distributions of the symmetrical three-beam interference in different polarization modes can be expressed as:

$$
\begin{aligned}
& I_{T E-T M-T E}=3 A^{2} \\
& +2 \cos \theta \cdot A^{2} \cdot \cos [k \cdot \sin \theta \cdot(x-y)] \\
& -2 \cos \theta \cdot A^{2} \cdot \cos [k \cdot \sin \theta \cdot(x+y)] \\
& -2 A^{2} \cdot \cos (2 k \cdot \sin \theta \cdot x)
\end{aligned}
$$

Figure 2 shows the simulation results via MATLAB. According to the results shown in Figures $2 a-b$, it is obvious that the simulated patterns are little different between TETE-TE mode and TM-TM-TM mode when the incident angles are $20^{\circ}$. In the case of TE-TE-TE mode, the maximum intensity is not affected by the incident beam angles from Eq. (6). It can be seen from Figures $2 c-d$ that there are identical distributions of dots in the TE-TM-TE mode and TM-TETM mode. From Eq. (8), theoretically, the maximum intensity is close to $7.5 \mathrm{~A}^{2}$ for the TE-TM-TE mode.
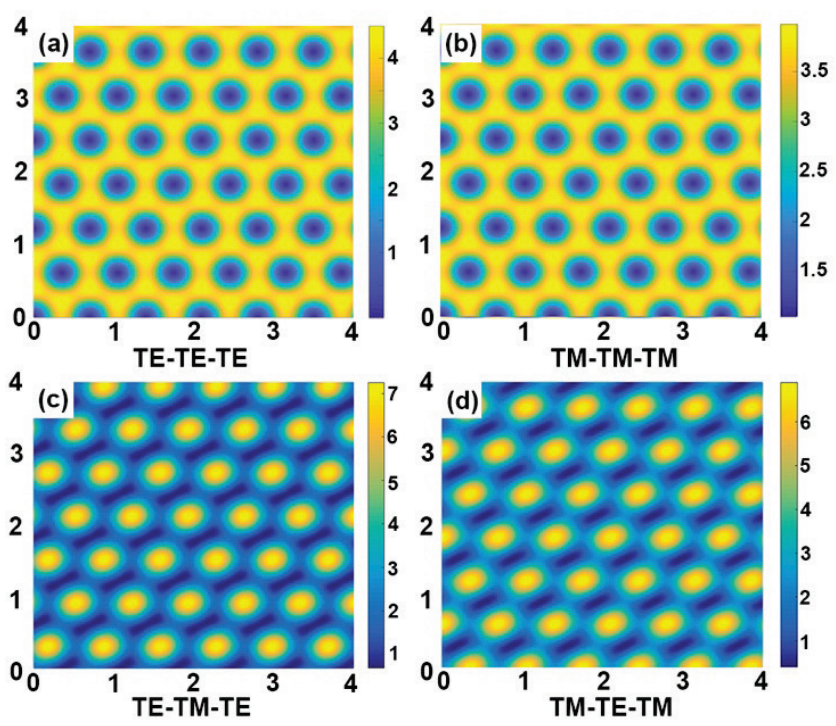

Fig. 2 Simulation results of symmetrical three-beam interference in $Z=0$ plane $\left(\theta=20^{\circ}\right)$.

In the asymmetrical three-beam interference (Figure 1b), there are three cases of polarization modes because two beams perpendicular to each other cannot interfere [29]. The polarization modes are TE-TM-TE, TM-TE-TM, and TMTM-TE. Table 2 shows their corresponding polarization vectors.

$$
\begin{aligned}
& I_{T M-T E-T M}=3 A^{2} \\
& -2 \cos \theta \cdot A^{2} \cdot \cos [k \cdot \sin \theta \cdot(x-y)] \\
& +2 \cos \theta \cdot A^{2} \cdot \cos [k \cdot \sin \theta \cdot(x+y)] \\
& -2\left(\cos ^{2} \theta-\sin ^{2} \theta\right) \cdot A^{2} \cdot \cos (2 k \cdot \sin \theta \cdot x)
\end{aligned}
$$




$$
\begin{aligned}
& I_{T M-T M-T E}=3 A^{2} \\
& +2 A^{2} \cdot \sin ^{2} \theta \cdot \cos [k \cdot \sin \theta \cdot(x-y)] . \\
& -2 A^{2} \cdot \cos \theta \cdot \cos [k \cdot \sin \theta \cdot(x+y)]
\end{aligned}
$$

Figure 3 shows the simulation results via MATLAB. In the asymmetrical three-beam interference, the maximum intensity is close to $9 \mathrm{~A}^{2}$ for the TE-TM-TE and TM-TE-TM modes at small incident angles. This indicates that the asymmetrical configuration with the azimuth angles of $0^{\circ}, 90^{\circ}$, and $180^{\circ}$ has a higher intensity ratio of optical distributions than the symmetrical three-beam interferences. Besides, the intensity of TM-TE-TM modes is more sensitive to the incident angle offset than that of TE-TM-TE mode, as shown in Figure 4 . The incident angles $(\theta)$ were changed to $45^{\circ}$ and $70^{\circ}$, respectively. Figures $3 \mathrm{~b}, 4 \mathrm{~b}$ and $4 \mathrm{~d}$ show that the pattern periods are changed. The interference patterns are obviously different. by:

The pattern periods along the $\mathrm{x}$ and $\mathrm{y}$ axes are computed

$$
\begin{aligned}
& d_{x}=\frac{\lambda}{\sin \theta_{1}+\sin \theta_{3}}=\frac{\lambda}{2 \sin \theta}, \\
& d_{y}=\frac{\lambda}{\sin \theta_{2}}=\frac{\lambda}{\sin \theta} .
\end{aligned}
$$

The above formulas clearly show the influence of the incident angle on the periods of patterns. $d_{y}$ in the y direction is independently changed by changing the incident angle of Beam 2 with the azimuth angle of $90^{\circ}$. In the TM-TM-TE mode (Figure 3c), the polarization directions of Beam 1 and Beam 3 (Figure $1 \mathrm{~b}$ ) are perpendicular to each other, and Beam 2 interferes with these two beams respectively and superimposed to form a chain-like pattern with a maximum intensity of $\approx 5 \mathrm{~A}^{2}$.
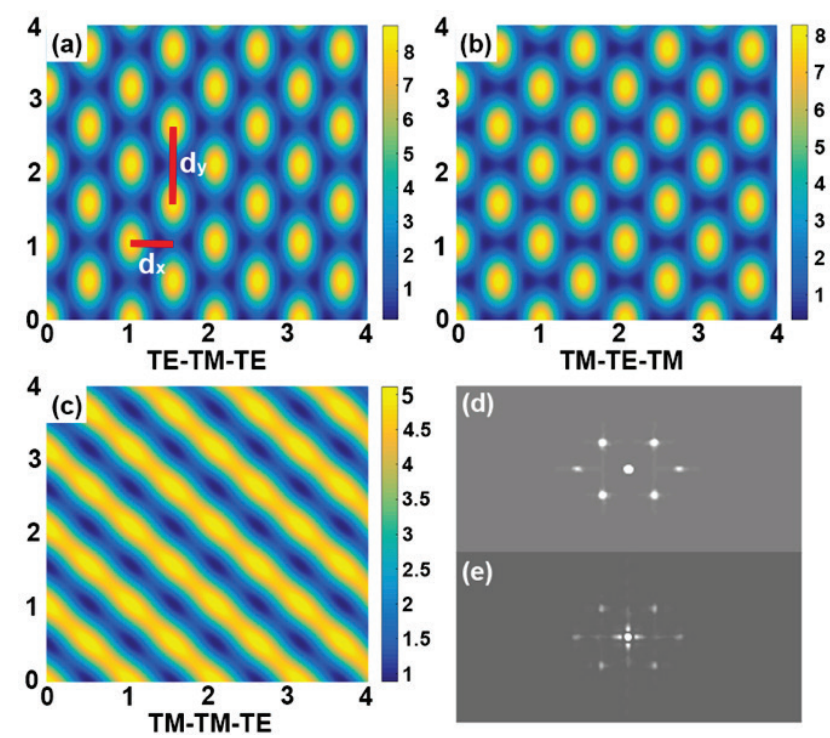

Fig. 3 (a-c) Simulation results of asymmetrical three-beam interference in $\mathrm{Z}=0$ plane $\left(\theta=20^{\circ}\right)$. (d-e) The frequency spectra of (a) and (b), respectively.
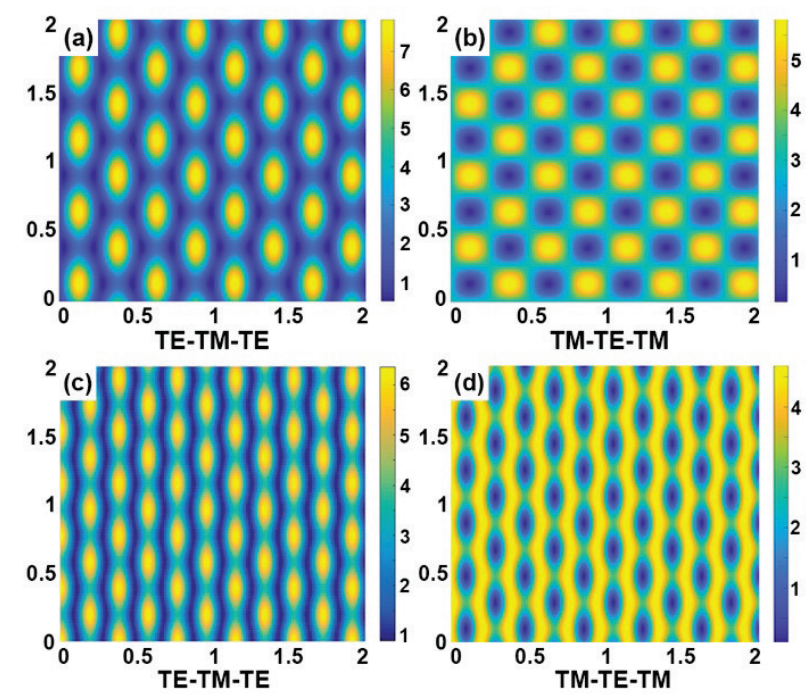

Fig. 4 Simulation results of asymmetrical three-beam interference with different incident angles in $\mathrm{Z}=0$ plane. (a-b) $\theta=45^{\circ}$; (c-d) $\theta$ $=70^{\circ}$.

In general, multi-beam interference can generate evenly distributed periodic patterns. However, in practice, amplitude modulation effects are almost unavoidably introduced in interferogram because of a misaligned azimuth angle [19] or unequal incident angles of coherent beams [30], which is not desired for many applications [32-34]. In the interference of three non-coplanar beams, a misaligned azimuth angle of coherent beams affects the distribution of dots, and the unequal incident angles can change the periods of the patterns. There is no doubt that the asymmetrical three-beam LIL avoids modulated interference effects.

\section{Experiment}

The asymmetric three-beam LIL system was designed and built, and Figure 5 schematically depicts the optical setup. The light source of the system is a diode-pumped solid-state laser (MSL-FN-360-S, CNI) with a wavelength of $360 \mathrm{~nm}$ and a power of $80 \mathrm{~mW}$. These beams were split via two beam splitters, quarter-wave plates and polarizers were used to precisely control the powers of the three beams and their polarization modes. The azimuth angles were $0^{\circ}$, $90^{\circ}$, and $180^{\circ}$, determined by the splitters. Three mirrors directly guided the incident angles of coherent beams. The incident angles were $13.5^{\circ}$. Therefore, the design of the optical path controlled the parameters of LIL. The power of the laser beams was measured with a photodiode power sensor (S120VC, Thorlabs).

The positive photoresist (AR-P 3740, Allresist) was spun on a silicon wafer at $4000 \mathrm{rpm}$ to produce a $1.4 \mu \mathrm{m}$ thick film, and was soft-baked for $1 \mathrm{~min}$ at $100^{\circ} \mathrm{C}$. After exposed to the LIL with the dose of $40-80 \mathrm{~mJ} \cdot \mathrm{cm}^{-2}$, the periodic structure was generated during the developing process in a developer (AR 47, Allresist) for $1 \mathrm{~min}$. After the cleaning and drying, the morphologies of the samples were characterized via the scanning electron microscope (SEM, Quanta250, FEI), as shown in Figures 6a-b. 


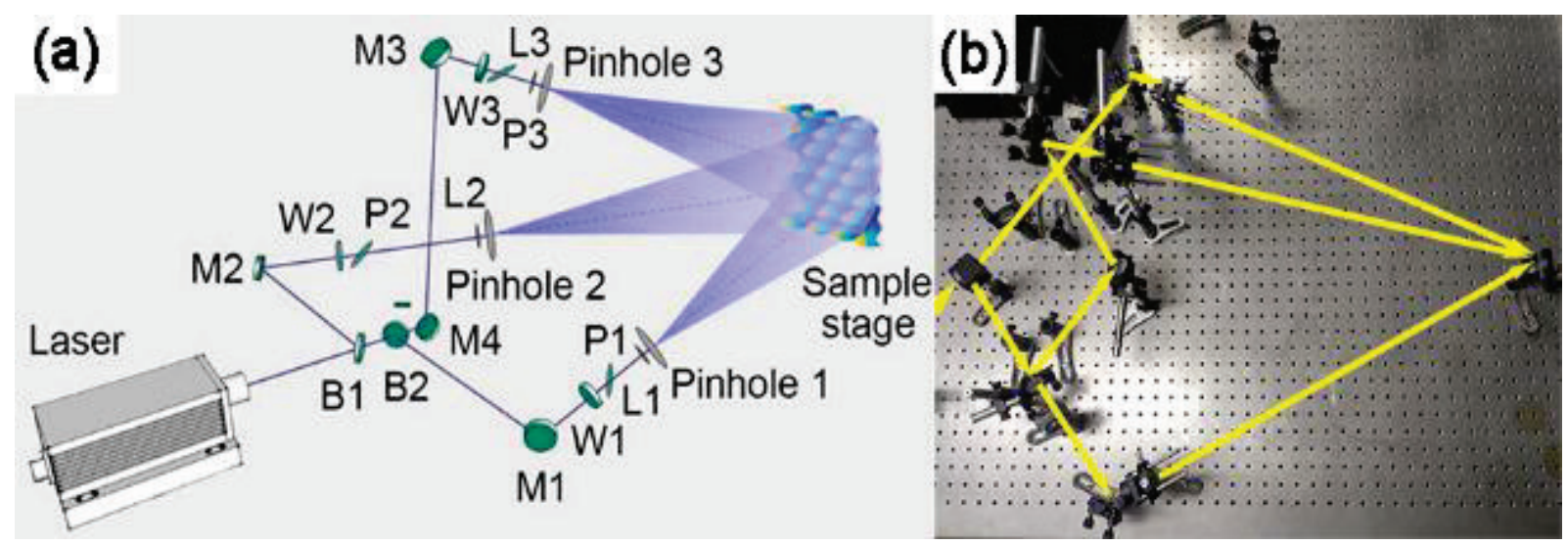

Fig. 5 Schematic diagram of the asymmetric three-beam LIL. (a) Schematic diagram: B, beam splitter; M, mirror; W, quarter-wave plate; P, polarizer; and L, lens. (b) Photo of the asymmetric three-beam LIL system.

From the SEM images, the experimentally obtained structures exhibit good agreement with the corresponding simulated results (Figures 3a-b). However, it is not enough to analyze the structure which is consistent from the spatial structures. FFT analysis converts a structure from its space domain to the frequency domain [35], so that we can accurately study features of the period structures fabricated via the asymmetric three-beam LIL.

As shown in Figures 6c-d, the frequency spectra show the frequency distributions of the periodic structures. Comparing the spectrogram of Figure 3d, it can be found that the frequency distribution of the structure (Figure 6a) and the frequency spectrum of the simulated pattern (Figure 3a) are basically the same. According to Equations (13) and (14), the theoretical periods of the interference pattern are $d_{x}=$ $0.771 \mu \mathrm{m}$ and $d_{y}=1.542 \mu \mathrm{m}$. From the SEM image (Figure $6 \mathrm{a})$, the periods of the structure in $\mathrm{x}$ and $\mathrm{y}$ directions are $0.771 \mu \mathrm{m}$ and $1.516 \mu \mathrm{m}$, respectively, measured by SEM. It means that the actual incident angle of Beam 2 is $0.233^{\circ}$ lager than the theoretical value. In the case of TM-TE-TM mode, there is a slight deviation between Figures $3 \mathrm{e}$ and $6 \mathrm{f}$, mainly because the incident angle of Beam 2 is slightly smaller than that of the other two beams in the asymmetric three-beam LIL system. From the SEM image (Figure 6b), the periods of the structure in the $\mathrm{x}$ and $\mathrm{y}$ directions are 0.747 $\mu \mathrm{m}$ and $1.557 \mu \mathrm{m}$, respectively. Compared with the theoretical analyses, the error of Beam 2's incident angle is $-0.132^{\circ}$, and error of Beam 1 and Beam 3's incident angle is $0.443^{\circ}$. The slightly different incident angels do not generate modulation interference patterns in either the TE-TM-TE or TMTE-TM mode, which further proves the asymmetrical threebeam can avoid the modulation effect.

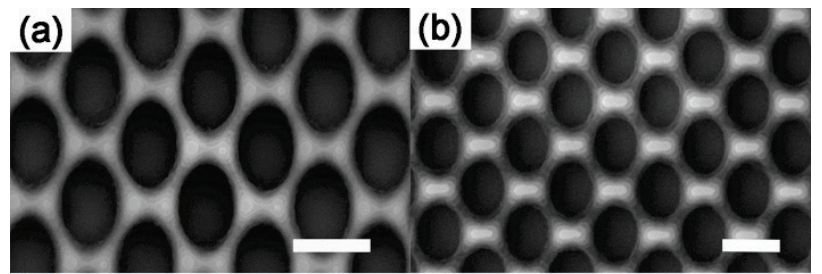

(c)

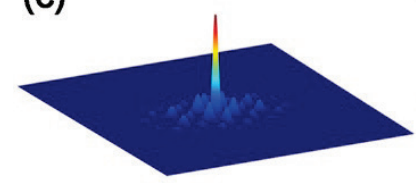

(e)

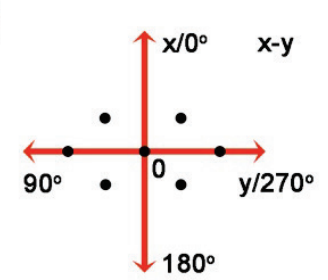

(d)

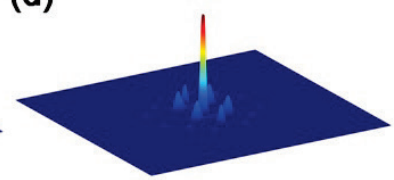

(f)

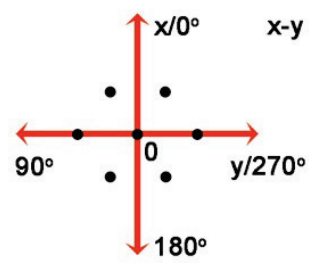

Fig. 6 SEM images of periodic structures fabricated by asymmetric three-beam LIL with the TE-TM-TE mode (a) and TM-TE-TM mode (b) and their frequency spectra (c-d), scale bar $=1 \mu \mathrm{m}$. (e) and (f) are the schematic diagrams of frequency spectra corresponding to (c) and (d), respectively.

The asymmetrical three-beam interference with the TETM-TE mode forms a higher intensity ratio of optical distribution compared to the symmetrical three-beam interference with the TE-TM-TE mode, as shown in Figure 7a, and the incident angles are $13.5^{\circ}$. The optical distributions were clearly recorded on photoresist. In comparison, the advantage of the asymmetrical three-beam interference is selfevident from the cross-section SEM images of structures (Figures $7 b-c$ ), which can effectively obtain structures with a higher duty cycle at the same radiation energy. This design of the asymmetrical optical path can also be used to laser interference patterning for directly processing on various metals and semiconductors. 

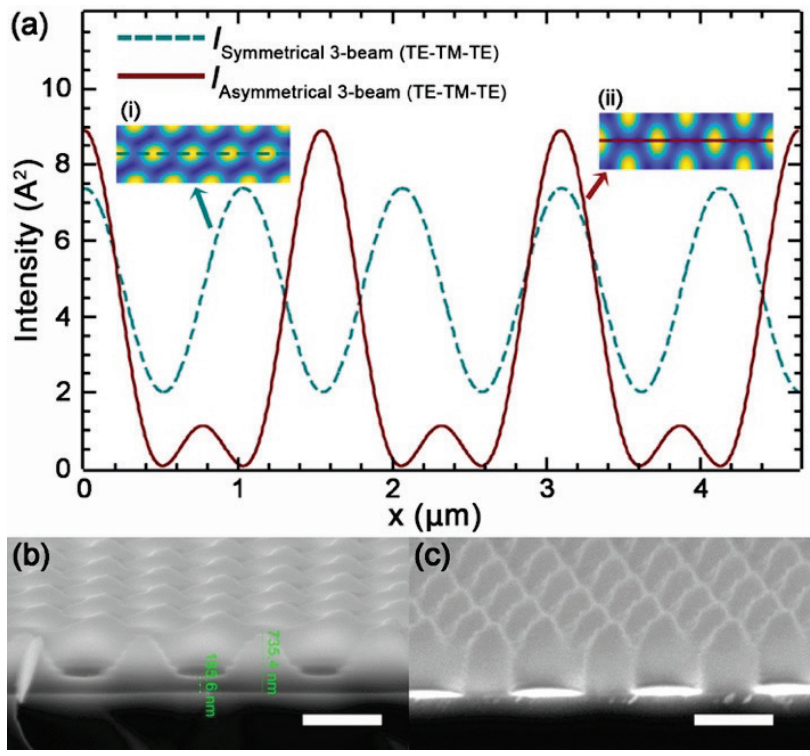

Fig. 7 (a) Intensity curves along the corresponding lines in the insets; the insets are optical patterns of the symmetrical 3-beam interference with the TE-TM-TE mode (i) and the asymmetrical 3beam interference with the TE-TM-TE mode (ii), respectively. (bc) The SEM images of the photoresist samples generated at the same exposure dose $\left(60 \mathrm{~mJ} \cdot \mathrm{cm}^{-2}\right)$ : (b) and (c) correspond to (i) and (ii), respectively. Scale bar $=1 \mu \mathrm{m}$.

\section{Conclusions}

In this work, we propose an asymmetrical design of three-beam LIL. The system is simple with respect to the design of optical paths, and is able to effectively control the formation of interference patterns so that makes it easier to fabricate the periodic structures with high duty cycles. The asymmetric three-beam interference $\left(0^{\circ}, 90^{\circ}, 180^{\circ}\right)$ with the TE-TM-TE mode can form interference patterns with a maximum intensity of $9 \mathrm{~A}^{2}$ which is higher than the symmetric three-beam interference $\left(0^{\circ}, 120^{\circ}, 240^{\circ}\right)$. The asymmetrical three-beam LIL system has been built, and the experiment results have exhibited good correspondence with the theoretical analyses and simulations, verified by SEM images and FFT analyses. The simple design of the multi-beam LIL has great advantages for fabricating periodic structures. The system construction is simple and it can effectively adjust various parameters for accurately controlling the features of periodic structures. In addition, the asymmetrical threebeam LIL also avoids modulation patterns in multi-beam LIL systems, and reduces the difficulty of processing because it can form an optical field distribution with a high intensity ratio as the exposed tool, which is used for lager-area fabrication of functional surfaces, such as structural colour [11-12], anti-reflection [16-17], super-hydrophobicity [3637], and surface-enhanced Raman scattering (SERS) [38-39].

\section{Acknowledgments and Appendixes}

This work was supported by EU H2020 Program (Grant NanoStencil No.767285), National Natural Science Foundation of China (Grant No.11504030, Grant No.11673082, Grant No.11103047), Jilin Provincial Science and Technology Program (Grant No.20190702002GH), and "111" Project of China (No.D17017).

\section{References}

[1] C. Zhang, D.A. Mcadams, and J.C. Grunlan: Adv. Mater., 28, (2016) 6292.

[2] Y. Si, Z. Dong, and L. Jiang: ACS Central Sci., 4, (2018) 1102.

[3] H.J. Choi, D. Huh, J. Jun, and H. Lee: Appl. Spectroscopy Rev., 54, (2019) 719.

[4] Z. Huang, N. Geyer, P. Werner, J. D. Boor, and U. Gösele: Adv. Mater., 23, (2011) 285.

[5] M. Altissimo: Biomicrofluidics, 4, (2010) 026503.

[6] A. Joshi-Imre and S. Bauerdick: J. Nanotechnol., 2014, (2014) 170415.

[7] G.Y. Liu, S. Xu, and Y. Qian: Accounts Chem. Res., 33, (2000) 457.

[8] M. Yang, Q. Wu, Z. Chen, B. Zhang, B. Tang, J. Yan, I. Drevensek-Olenik, and J. Xu: Opt. Lett., 39, (2014) 343.

[9] D. Serien and K. Sugioka: Opto-Electron. Adv., 1, (2018) 180008.

[10]H. Tao and J. Lin: Opt. Laser. Eng., 114, (2019) 31.

[11] R.H. Siddique, R. Hünig, A. Faisal, U. Lemmer, and H. Hölscher: Opt. Mater. Express, 5, (2015) 996.

[12]H. Wu, Y. Jiao, C. Zhang, C. Chen, L. Yang, J. Li, J. Ni, Y. Zhang, C. Li, Y. Zhang, S. Jiang, S. Zhu, Y. Hu, D. $\mathrm{Wu}$, and J. Chu: Nanoscale, 11, (2019) 4803.

[13]D. Xia and S.R.J. Brueck: Nano Lett., 8, (2008) 2819.

[14] Y.L. Yang, C.C. Hsu, T.L. Chang, L.S. Kuo, and P.H. Chen: Appl. Surf. Sci., 256, (2010) 3683.

[15] S.G. Park, J.H. Moon, S.K. Lee, J. Shim, and S.M. Yang: Langmuir., 26, (2009) 1468.

[16] L. Zhao, Z. Wang, J. Zhang, L. Cao, L. Li, Y. Yue, and D. Li: Appl. Surf. Sci., 346, (2015) 574.

[17]L. Dong, Z. Zhang, L. Wang, Z. Weng, M. Ouyang, Y. $\mathrm{Fu}$, J. Wang, D. Li, and Z. Wang: Appl. Optics, 58, (2019) 6706

[18] Y. Hu, Z. Wang, Z. Weng, M. Yu, and D. Wang: Appl. Optics., 55, (2016) 3226.

[19]D. Wang, Z. Wang, Z. Zhang, Y. Yue, D. Li, R. Qiu, and C. Maple: J. Appl. Phys., 115, (2014) 233101.

[20]Z. Zhang, Z. Wang, D. Wang, and Y. Ding: J. Laser Appl., 26, (2014) 012010.

[21] X. Wang, J. Xu, H. Su, Z. Zeng, Y. Chen, and H. Wang: Appl. Phys. Lett., 82, (2003) 2212.

[22] S. Behera, M. Kumar, and J. Joseph: Optics Lett., 41, (2016) 1893.

[23] J. Xu, Z. Wang, Z. Zhang, D. Wang, and Z. Weng: J. Appl. Phys., 115, (2014) 203101.

[24]P. Graus, T.B. Möller, P. Leiderer, J. Boneberg, and N.I. Polushkin: Opto-Electron. Adv., 3, (2020) 190027.

[25] L. Wang, L. Dong, L. Li, Z. Weng, H. Xu, M. Yu, and Z. Wang: J. Mater. Sci., 53, (2018) 3239.

[26] S. Masui, Y. Torii, M. Michihata, K. Takamasu, and S. Takahashi: Opt. Express, 27, (2019) 31522.

[27]C. Tan, C.S. Peng, V.N. Petryakov, Y.K. Verevkin, J. Zhang, Z. Wang, S.M. Olaizola, T. Berthou, S. Tisserand, and M. Pessa: New J. Phys., 10, (2008) 023023.

[28] J.H. Moon, J. Ford, and S. Yang: Polym. Advan. Technol., 17, (2006) 83.

[29] C.W. Jui, A.J.C. Trappey, and C.C. Fu: Recent Pat. Nanotech., 12, (2018) 231. 
[30]Z. Zhang, L. Dong, Y. Ding, L. Li, Z. Weng, and Z. Wang: Opt. Express, 25, (2017) 29135.

[31] J. Zhang, Z. Wang, X. Di, L. Zhao, and D. Wang: Appl. Optics, 53, (2014) 6294.

[32] T. Tavera, N. Pérez, A. Rodríguez, P. Yurrita, S. M. Olaizola, and E. Castaño: Appl. Surf. Sci. 258, (2011) 1175.

[33] Y. Zabila, M. Perzanowski, A. Dobrowolska, and M. Marszałek: Acta Phys. Pol. A, 115, (2009) 591.

[34]C. Tan, C. S. Peng, V. N. Petryakov, Y. K. Verevkin, J. Zhang, Z. Wang, S. M. Olaizola, T. Berthou, S. Tisserand, and M. Pessa: New J. Phys., 10, (2008) 023023.

[35] M. Liu, L. Dong, Z. Zhang, L. Li, L. Wang, Z. Song, Z. Weng, X. Han, L. Zhou, and Z. Wang: Appl. Phys. Express, 12, (2019) 096503.

[36]L. Zhao, Z. Wang, J. Zhang, L. Cao, L. Li, Y. Yue, and D. Li: Appl. Surf. Sci., 346, (2015) 574.

[37]L. Dong, Z. Zhang, R. Ding, L. Wang, M. Liu, Z. Weng, Z. Wang, and D. Li: Surf. Coat. Tech., 372, (2019) 434.

[38]L. Wang, Z. Wang, L. Li, J. Zhang, J. Liu, J. Hu, X. Wu, Z. Weng, X. Chu, J. Li, and Z. Qiao: RSC Adv., 10, (2020) 2661.

[39] H.J. Ahn, P. Thiyagarajan, L. Jia, S.I. Kim, J.C. Yoon, E.L. Thomas, and J.H. Jang: Nanoscale, 5, (2013) 1836.

(Received: May 29, 2020, Accepted: July 27, 2020) 